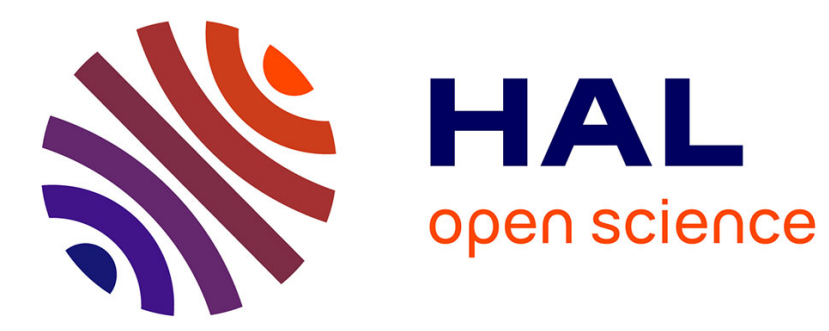

\title{
Contribution à l'étude de l'oxydation du niobium
}

\author{
D. Mollimard, J. Bardolle, D. Simon, C. Perrin, G. Dalibard
}

\section{To cite this version:}

D. Mollimard, J. Bardolle, D. Simon, C. Perrin, G. Dalibard. Contribution à l'étude de l'oxydation du niobium. Revue de Physique Appliquée, 1970, 5 (3), pp.599-608. 10.1051/rphysap:0197000503059900 . jpa-00243432

\section{HAL Id: jpa-00243432 https://hal.science/jpa-00243432}

Submitted on 1 Jan 1970

HAL is a multi-disciplinary open access archive for the deposit and dissemination of scientific research documents, whether they are published or not. The documents may come from teaching and research institutions in France or abroad, or from public or private research centers.
L'archive ouverte pluridisciplinaire HAL, est destinée au dépôt et à la diffusion de documents scientifiques de niveau recherche, publiés ou non, émanant des établissements d'enseignement et de recherche français ou étrangers, des laboratoires publics ou privés. 


\title{
CONTRIBUTION A L'ÉTUDE DE L'OXYDATION DU NIOBIUM
}

\author{
par Messieurs D. MOLlimaRD, J. BARDOLLE, D. SIMON, C. PERRIN et G. DALIBARD
}

\begin{abstract}
Résumé. - Le présent travail est une étude de l'oxydation du Niobium dans diverses conditions de température et pression sous ses aspects morphologique, cinétique et cristallographique.

Les résultats obtenus concernent tout d'abord la cinétique et l'anisotropie de formation des films minces interférentiels à $350^{\circ} \mathrm{C}$ et 10 torrs d'oxygène. On observe des vitesses croissant dans l'ordre (100), (211), (111).

Aux basses pressions $\left(10^{-4}\right.$ à $10^{-6}$ torr) et températures élevées $\left(550\right.$ à $\left.950^{\circ} \mathrm{C}\right)$, la cinétique révèle deux mécanismes d'adsorption de l'oxygène avec des énergies d'activation de 3 et 26 kilocalories.

La formation de germes d'oxyde a été précisée. Les couches d'épaisseur notables obtenues aux températures élevées et à des pressions de l'ordre de 5 torrs ont été également étudiées.

La nature des oxydes formés dans diverses conditions a été déterminée par diffractométrie $\mathrm{X}$.

Abstract. - In the present work, Niobium oxidation is studied in various conditions of temperature and pressure with respect to morphology, kinetics, and crystallographical aspects of the reaction.

At first, results were obtained in regard to kinetics and anisotropy of growth of thin films at $350^{\circ} \mathrm{C}$ and 10 torrs of oxygen pressure. Reaction velocities obtained are in the order (100), (211), (111).

At low pressures $\left(10^{-4}\right.$ to $10^{-6}$ torr) and high temperatures $\left(550\right.$ to $\left.950^{\circ} \mathrm{C}\right)$, kinetics shows two adsorption mechanisms of the oxygen with activation energies of 3 and 26 kilocalories.

Nucleation phenomenon is also studied with some details.

The growth of oxide films of noticeable thickness at high temperatures and pressures of the order of 5 torrs is also investigated.

The nature of oxide films obtained in various conditions was determined by X Ray Diffractometry.
\end{abstract}

Introduction. - Le présent travail concerne un ensemble de recherches sur contrat DRME avec du niobium de haute pureté* fourni par la Société ImphyKuhlmann. Ces recherches sont relatives aux aspects suivants de la réaction d'oxydation :

$1^{0}$ Formation de films minces interférentiels à température moyenne.

$2^{\circ}$ Adsorption de l'oxygène aux températures élevées et basses pressions.

$3^{\circ}$ Formation de germes d'oxyde également aux températures élevées et aux basses pressions.

$4^{0}$ Couches continues d'épaisseur notable.

Les divers aspects morphologique, cinétique et cristallographique ont été envisagés. Une étude aux rayons $\mathrm{X}$ a été réalisée sur les produits de la réaction dans les diverses conditions choisies.

Nous insisterons peu ici sur les appareillages utilisés, mais davantage sur les résultats.

La préparation des échantillons comporte successivement un polissage aux papiers abrasifs, une recristallisation sous vide à $10^{-7}$ torr et $1000^{\circ} \mathrm{C}$ qui dure généralement $24 \mathrm{~h}$, suivie d'un polissage électrolytique et d'un recuit final sous $10^{-7}$ torr à $1000^{\circ} \mathrm{C}$, d'une durée de $24 \mathrm{~h}$.

Bien que les divers points abordés puissent paraître parfois éloignés des préoccupations des utilisateurs futurs de ce métal, nous pensons qu'une connaissance approfondie des problèmes fondamentaux de la réaction avec l'oxygène ou d'autres gaz est indispensable pour bien comprendre les mécanismes qui entrent en jeu et pour apporter si possible une protection réellement efficace contre l'oxydation.

I. Formation des films minces d'oxyde: - ANISOTROPIE RÉACTIONNELLE. - La détermination d'un état de surface convenable a conduit à l'étude de la formation de films minces d'oxyde présentant des teintes d'interférence. L'appareillage utilisé qui ne sera pas décrit ici en détail permet de réaliser soit un chauffage sous vide, soit une oxydation sous oxygène pur à diverses températures.

$$
\begin{array}{cccccccccc}
\mathrm{C} & \mathrm{N} & \mathrm{O} & \mathrm{Ta} & \mathrm{W} & \mathrm{Fe} & \mathrm{Ni} & \mathrm{Cr} & \mathrm{Mo} & \mathrm{Zr} \\
45 & 21 & 55 & 35 & 20 & <10 & <10 & <5 & <20 & <50 \\
& & & & & & & & \\
\mathrm{Sn} & \mathrm{Al} & \mathrm{Si} & \mathrm{Ti} & \mathrm{V} & \mathrm{B} & \mathrm{As} & \mathrm{P} & \mathrm{S} \\
<20 & <30 & <7 & <20 & <10 & <2 & <1 & <20 & <10
\end{array}
$$

De nombreux essais ont eu pour objet de déterminer les conditions précises dans lesquelles s'effectue la réaction. Nous commencerons par décrire l'aspect qualitatif des phénomènes en insistant sur l'influence de l'orientation cristalline.

Lorsqu'on effectue la réaction sur échantillons polycristallins à $350^{\circ} \mathrm{C}$ pendant $2 \mathrm{~h}$ environ sous 
10 torr d'oxygène on constate que la surface est recouverte d'un film interférentiel dont la couleur est fonction de l'orientation des cristaux sous-jacents.

$\mathrm{Au}$ forts grossissements du microscope optique on note que des germes apparaissent également après un temps suffisant à l'intérieur ou à la surface de ces films interférentiels. Nous reviendrons plus loin sur cette question.

L'anisotropie réactionnelle est donc très nette. Nous avons pu vérifier que la vitesse de réaction dans les conditions indiquées est maximale sur le plan (111). Cette vitesse croît dans l'ordre suivant : (100), (211), (111). Il semble qu'à $350^{\circ} \mathrm{C}$ les vitesses restent voisines pour les plans (111), (110) et (211).

Il est en tout cas certain que le plan (100) qui correspond à une face du cube de la structure cubique centrée du niobium, est celui de réactivité minimale dans les conditions indiquées.

Il convient de signaler que cette étude a pu être réalisée en utilisant des cristaux uniques de grandes dimensions et d'orientations variées qui étaient déterminées par la méthode de Laue en retour.

Une étude qualitative effectuée à température plus basse, $340^{\circ} \mathrm{C}$ et 10 torr nous a révélé une plus grande différenciation des vitesses de réaction sur les orientations (111), (110) et (2 11). Il a donc paru nécessaire de réaliser une étude cinétique en fonction de la température et de la pression.

2. Etude CINÉTIQUE. - Bien que les recherches sur cristaux uniques soient déjà avancées nous nous contenterons actuellement de décrire les travaux qui ont été réalisés sur la cinétique de croissance de ces films sur échantillons polycristallins.

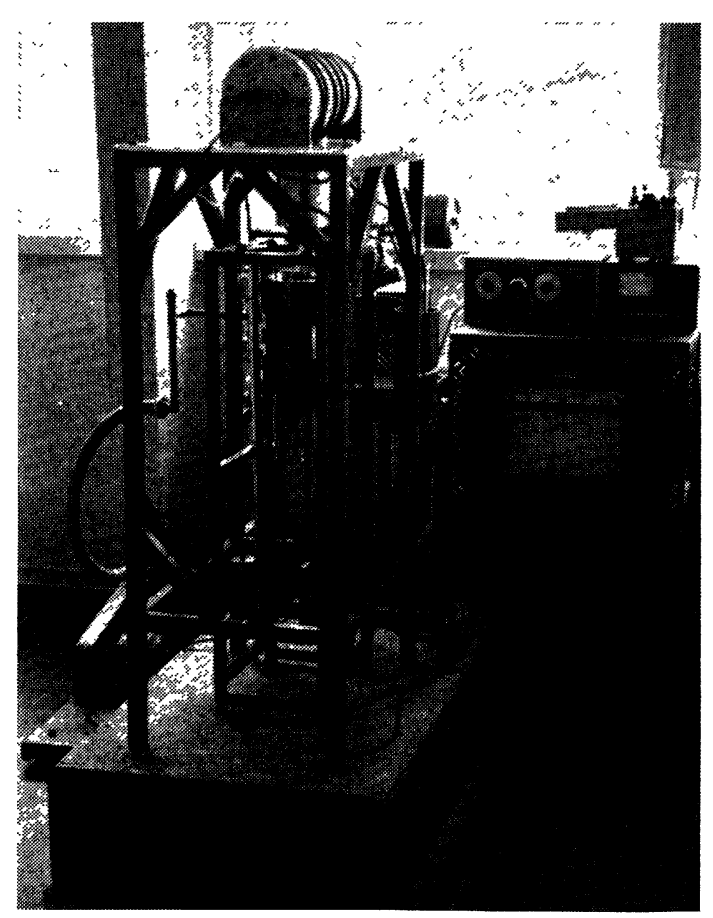

FIG. 1. - Appareil pour l'étude microgravimétrique des films interférentiels d'oxyde de niobium.
La figure 1 est une vue de l'appareil qui a été construit pour cette étude et qui comporte une balance de Cahn pour laquelle les conditions d'élimination des vibrations ont été particulièrement poussées. On notera que son implantation a été réalisée sur un bloc en béton isolé du sol par une couche épaisse de liège. Toutes ces précautions ont été nécessaires afin d'atteindre une sensibilité de 0,2 microgrammes qui permet en théorie d'apprécier une épaisseur du film d'oxyde d'environ 10 Angströms par $\mathrm{cm}^{2}$ de surface réactionnelle.

La figure 2 montre les courbes cinétiques obtenues sous une pression de 75 torr d'oxygène à 300,350 ,

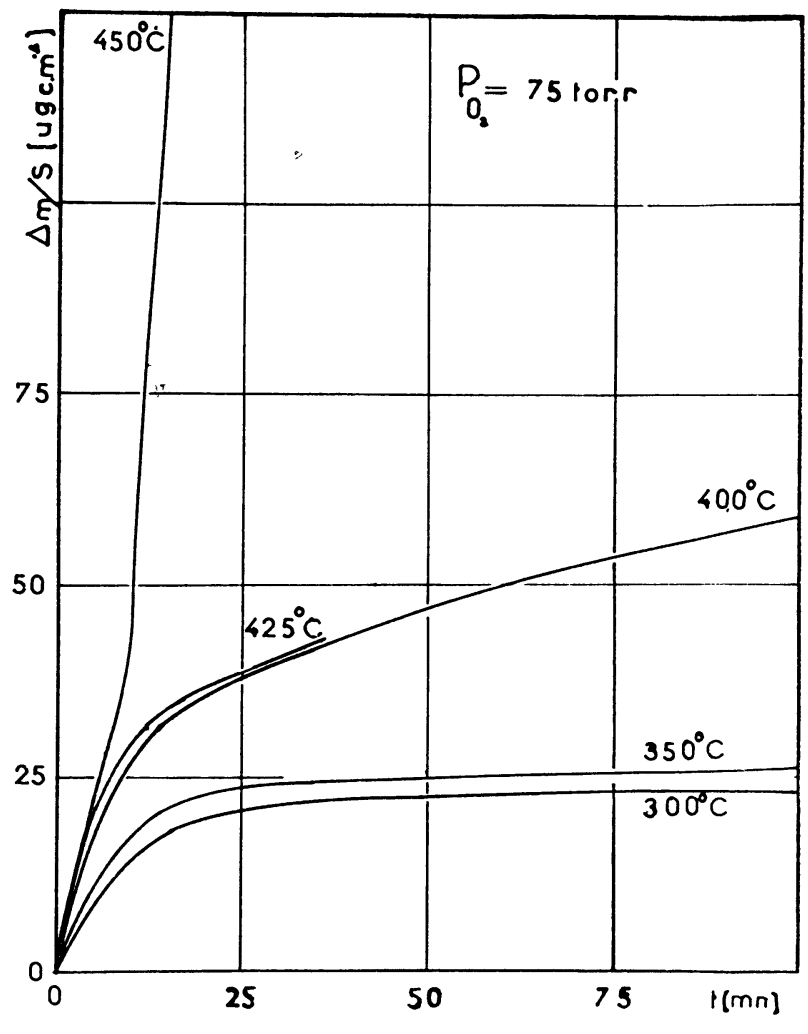

FIG. 2.

400,425 et $450^{\circ} \mathrm{C}$. On a porté ici la prise de masse par unité de surface $\Delta m / S$ en microgramme par $\mathrm{cm}^{2}$ en fonction du temps en minutes. On notera la vitesse beaucoup plus importante du phénomène à $450^{\circ} \mathrm{C}$.

L'analyse des courbes montre que la cinétique est complexe et lorsqu'on porte $\log (\Delta m / S)$ en fonction de $\log t$ on peut noter plusieurs étapes de la réaction.

Première étape. - Pendant les premières minutes la cinétique globale suit une loi de la forme $\Delta m / S=k t^{n}$ avec les valeurs suivantes de $n$ :

$$
\begin{aligned}
& \text { à } 350^{\circ} \mathrm{C}: n=0,853 \\
& \text { à } 400^{\circ} \mathrm{C}: n=0,866 \\
& \text { à } 450^{\circ} \mathrm{C}: n=0,855 .
\end{aligned}
$$

On voit que le coefficient $n$ trouvé est voisin de 1 et que par conséquent on se rapproche très nettement 
d'une loi linéaire dans les premiers instants de la réaction. Le calcul de l'énergie d'activation montre que celle-ci est relativement faible et voisine de $6 \mathrm{kcal} / \mathrm{mole}$. On a donc ici vraisemblablement une réaction d'interface, légèrement freinée.

Deuxième étape. - Cette seconde partie des courbes paraît correspondre à une transition entre deux mécanismes. Elle est de type parabolique et liée vraisemblablement à une diffusion de l'oxygène dans le métal. Elle ne dure que quelques minutes aux températures considérées.

Troisième étape. - Une troisième étape suit les deux précédentes et répond à une loi de croissance logarithmique, actuellement en cours d'étude détaillée.

Il semble bien que la seconde et la troisième étape correspondent à l'apparition des films interférentiels visibles sur la surface de l'échantillon.

Quatrième étape. - Après un certain temps une accélération de la réaction se produit en même temps que des germes apparaissent à la surface du film d'oxyde. La réaction progresse alors très rapidement. L'étude de cette quatrième étape sur le plan cinétique est actuellement en cours.

Il faut bien noter que les courbes cinétiques obtenues ne donnent qu'un aspect global des phénomènes et en particulier, au moment où les teintes apparaissent, deux phénomènes simultanés ont également lieu, l'un étant la dissolution de l'oxygène dans le métal et l'autre la formation d'un film interférentiel. Il est donc nécessaire de distinguer ces deux phénomènes et dans ce but deux techniques sont envisagées: la méthode ellipsométrique et l'activation des échantillons aux neutrons. Un ellipsomètre permettra de suivre uniquement la cinétique de croissance du film superficiel. De son côté l'activation aux neutrons conduira à distinguer entre le film formé à la surface de l'échantillon et l'oxygène ayant pénétré dans le métal. Nous pensons pouvoir ainsi élucider de façon complète le mécanisme de cette réaction complexe.

Il faut signaler que des résultats très intéressants avaient déjà été obtenus par Hurlen [1] mais avec des différences notables avec nos propres résultats probablement par suite de la différence de préparation des échantillons.

3. NATURE DES FILMS FORMÉS. - Le passage au diffractomètre à Rayons $X$ d'échantillons oxydés vers $350^{\circ} \mathrm{C}$ pendant $1 \mathrm{~h} 30$ à une pression de quelques $\mathrm{mm}$ de mercure et présentant des teintes d'interférence ne donne pas de diagramme de Rayons X. Il n'a pas non plus été possible de mettre en évidence une structure cristallisée par diffraction des électrons: Il semble donc que l'oxyde formé soit amorphe ou mal cristallisé au moins au début de la réaction.

Par contre diverses expériences effectuées entre $304^{\circ} \mathrm{C}$ et $324^{\circ} \mathrm{C}$ pendant des temps variables de 24 à $112 \mathrm{~h}$ ont permis de mettre en évidence le sous-oxyde $\mathrm{NbO}_{x}$ qui est une phase quadratique dont la structure est en relation avec celle du métal : $a=3,39 \AA$, $c=3,27 \AA$ (paramètre de $\mathrm{Nb} 3,30 \AA$ ).

Un échantillon oxydé $100 \mathrm{~h}$ à $350^{\circ} \mathrm{C}$ et une pression d'oxygène égale à $250 \mathrm{~mm}$ de mercure présente des teintes d'interférence et sur ces teintes des germes interférentiels. Nous donnons dans le tableau I les raies obtenues au diffractomètre comparées aux raies de $\mathrm{NbO}_{x}$ données par Norman [2].

Nous avons indiqué la présence de germes formés à la surface des films interférentiels. Ces germes à partir de $380^{\circ} \mathrm{C}$ environ apparaissent sous forme d'oxyde blanc qui peut être reconnu comme du $\mathrm{Nb}_{2} \mathrm{O}_{5}$. On sait que cet oxyde présente d'assez nombreuses variétés et nous reviendrons sur ce problème au moment de l'étude des couches épaisses. Indiquons que les raies

TABLeAU I

$\left(\mathrm{NbO}_{x}\right)$

\begin{tabular}{|c|c|c|c|c|c|}
\hline \multicolumn{3}{|c|}{ Nos résultats } & \multicolumn{3}{|c|}{ NoRMaN [2] } \\
\hline $2 \theta$ & I mesurée & $d$ observée & $d$ observée & $d$ calculée & I (intensité) \\
\hline - & - & - & - & - & - \\
\hline 37,80 & f & 2,39 & 2,394 & 2,40 & 5 \\
\hline 38,30 & $\mathrm{~F}$ & 2,35 & 2,350 & 2,357 & 10 \\
\hline 54,30 & $\mathbf{M}$ & 1,695 & 1,693 & 1,697 & 4 \\
\hline 56,10 & $\mathrm{~F}$ & 1,631 & 1,636 & 1,637 & 2 \\
\hline $65,10-65,30$ & $\mathbf{M}$ & $1,43-1,42$ & 1,42 & & 2 \\
\hline 68,30 & $\mathrm{f}$ & 1,37 & 1,374 & 1,368 & 7 \\
\hline 69,30 & - & 1,35 & 1,352 & 1,353 & 5 \\
\hline \multirow{2}{*}{$95,50 \pm 0,2$} & \multirow{2}{*}{ - } & \multirow[t]{2}{*}{1,066} & \multirow{2}{*}{1,07} & 1,063 & 4 \\
\hline & & & & 1,060 & 4 \\
\hline 94 & - & 1,035 & 1,039 & 1,039 & 4 \\
\hline \multicolumn{6}{|l|}{$\mathrm{f}=$ faible } \\
\hline$F=$ forte & & & & & \\
\hline $\mathbf{M}=$ moyen & & & & & \\
\hline
\end{tabular}


observées pour un échantillon oxydé à $420^{\circ} \mathrm{C}$ pendant $1 \mathrm{~h} 30$ et sous une pression d'oxygène de $70 \mathrm{~mm}$ de mercure indiquent vraisemblablement la présence d'oxyde monoclinique $\mathrm{Nb}_{2} \mathrm{O}_{5} \alpha$ selon la notation de Goldschmidt. On a les raies suivantes :

$\begin{array}{cc}2 \theta & d \text { observés } \\ - & - \\ 22,60 & 3,93 \\ 25,85 & 3,46 \\ 28,42 & 3,14 \\ 42,80 & 2,12\end{array}$

Les trois premières raies pourraient également appartenir à une phase $\delta$ faiblement cristallisée et pseudo-hexagonale. Toutefois l'existence de cette phase proposée par Kofstad et également Terao, entre $350^{\circ} \mathrm{C}$ et $600^{\circ} \mathrm{C}$ a été mise en doute par d'autres auteurs. Aussi il semble plus vraisemblable d'admettre essentiellement la présence d'oxyde $\alpha$ auquel correspond à coup sûr la dernière raie indiquée ci-dessus.

II. Adsorption de l'oxygène aux températures élevées et aux basses pressions. - 1 . Appareillage. - Afin de réaliser l'étude de l'adsorption de l'oxygène aux températures élevées un appareil a été construit qui permet de suivre la réaction et ceci à des pressions comprises entre $10^{-2}$ et $10^{-5}$ torr, avec une sensibilité de l'ordre du microgramme.

Pour ce faire, une balance de Cahn a été utilisée à laquelle est relié un tube en silice transparente de grand diamètre (de l'ordre de $5 \mathrm{~cm}$ ) et disposé verticalement. Il est possible de faire le vide dans ce tube ou d'obtenir à l'aide d'une fuite réglable les pressions indiquées ci-dessus. Une jauge à ionisation est placée à chaque extrémité du tube et les pressions mesurées permettent de se rendre compte qu'avec les dispositifs utilisés le débit gazeux est d'environ 281 par seconde.

Il peut être chauffé à la température désirée à l'aide d'un four Chevenard à tige dilatable. La température est mesurée directement à l'aide d'un couple thermoélectrique placé dans un doigt de gant au voisinage de l'échantillon.

La figure 3 donne un schéma de cet appareillage.

2. Résultats obtenus. - Avant de décrire l'ensemble de nos résultats, il convient de citer un certain nombre de travaux antérieurs. Indiquons que la réaction avait été étudiée sous basse pression d'oxygène en particulier par Inouye [3] entre $3 \times 10^{-5}$ et $5 \times 10^{-3}$ torr pour les températures de 850,1000 et $1250^{\circ} \mathrm{C}$ et par Kofstad et Espevik [4] entre 1200 et $1700^{\circ} \mathrm{C}$.

Pour notre part nous avons étudié l'oxydation du Niobium entre 550 et $950^{\circ} \mathrm{C}$. L'ensemble des résultats peut être décrit en fonction des facteurs température, pression et orientation cristalline.

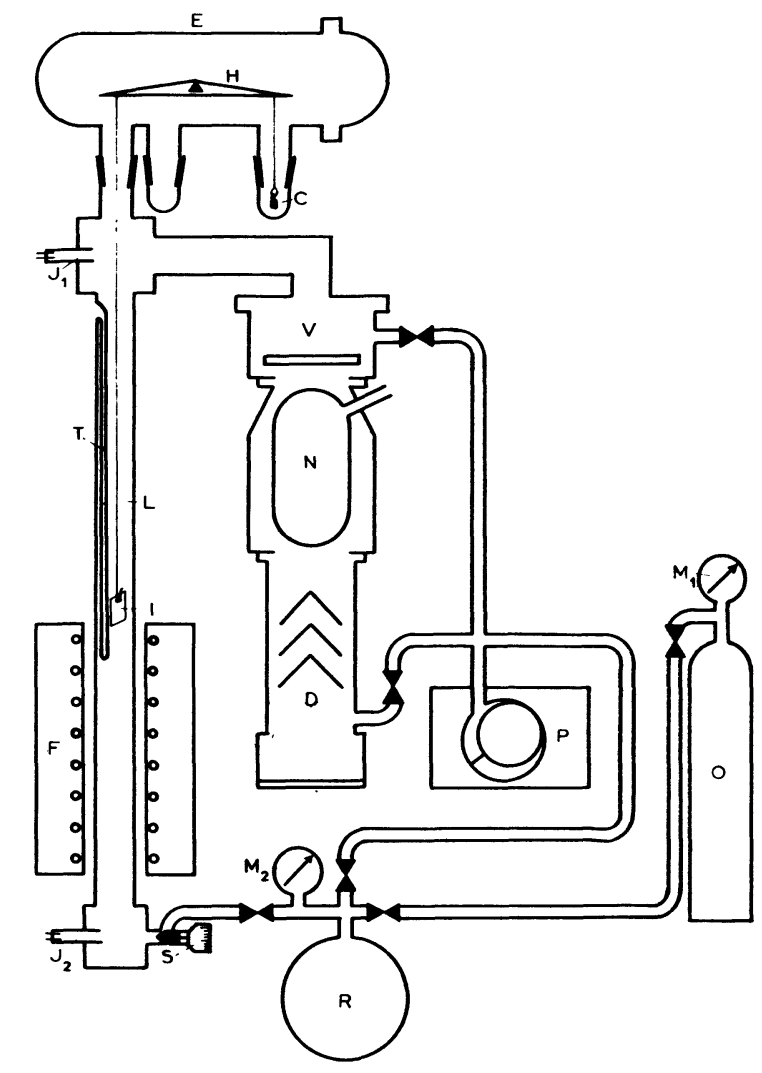

Fig. 3. - Schéma sur l'appareillage d'oxydation du niobium aux basses pressions d'oxygène.

$\mathrm{O}=$ réserve d'oxygène sous pression,

$\mathbf{M}_{1}=$ manomètre détendeur,

$R$ = réserve d'oxygène sous basse pression (50 à 100 torr),

$\mathbf{M}_{2}=$ manomètre,

$\mathbf{P}=$ pompe à palettes,

D = pompe à diffusion d'huile,

$\mathbf{N}=$ piège à azote liquide,

$\mathrm{V}$ = vanne baffle,

$\mathrm{E}=$ enceinte de la microbalance (pyrex),

$\mathbf{H}=$ microbalance,

C $=$ contrepoids,

I = échantillon,

L = tube laboratoire (quartz),

$\mathrm{F}=$ four à résistance,

$\mathbf{T}=$ thermocouple,

$\mathrm{S}=$ fuite réglable pour l'introduction de l'oxygène,

$\mathbf{J}_{1}$ et $\mathbf{J}_{2}=$ jauges à ionisation.

a. Influence de la température. - La figure 4 montre les courbes cinétiques obtenues avec des échantillons polycristallins aux températures suivantes : 550 , $600,650,700,850,900,950$ et $1000^{\circ} \mathrm{C}$ et sous une pression d'oxygène de $9 \times 10^{-5}$ torr. On a porté en ordonnée les accroissements de poids par unité de surface $\Delta m / S$ en microgramme par $\mathrm{cm}^{2}$ en fonction du temps en minute. On peut constater que les lois obtenues sont toutes linéaires : $\Delta m / S=k t$.

La figure 5 représente la courbe $\log k$ en fonction de $1 / T$ pour diverses températures. On notera que les points obtenus s'alignent sur deux droites distinctes, l'une à plus basse température correspond à une énergie d'activation pour la réaction $\mathrm{W}_{2}=26 \mathrm{kcal} / \mathrm{mole}$, l'autre à température plus élevée à une énergie d'acti- 


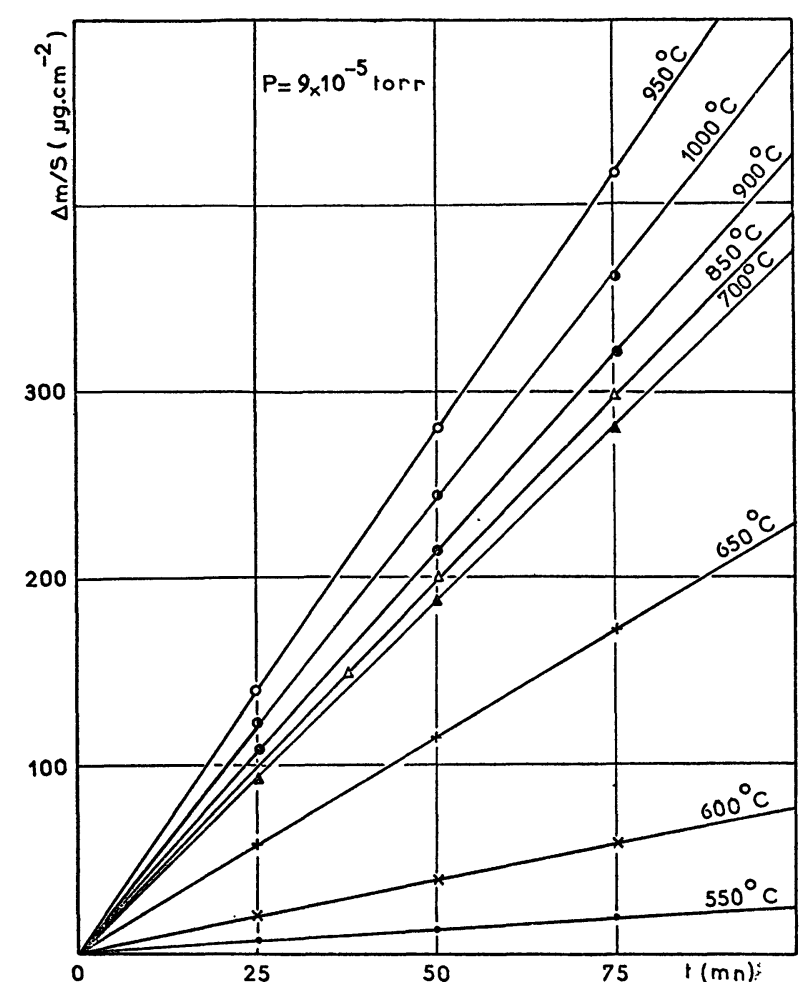

FIG. 4.

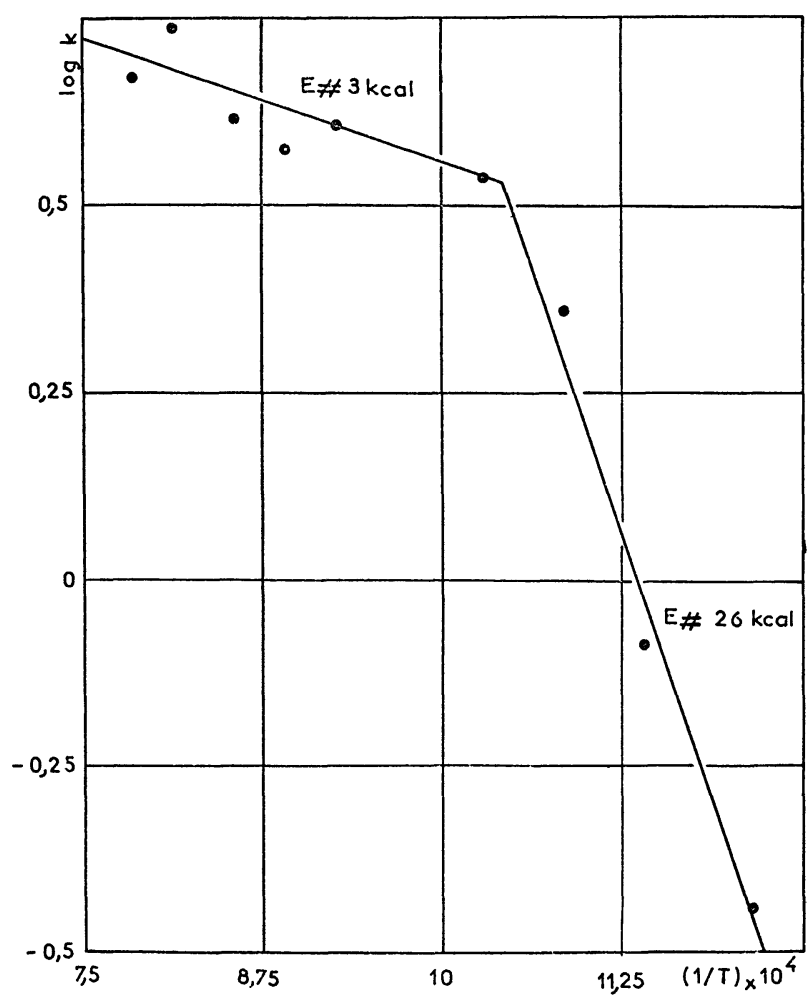

Fig. 5.

vation $W_{1}$ de $3 \mathrm{kcal} /$ mole. La préparation des échantillons joue un rôle essentiel dans les résultats obtenus et les recuits sous vide doivent s'effectuer de telle façon que les échantillons restent disposés verticale- ment sans qu'aucune de leurs faces soit en contact avec un support même en Niobium.

Nous reviendrons plus loin sur l'interprétation de ces résultats.

b. Influence de la pression. - L'influence de la pression a été étudiée à une même température, $850^{\circ} \mathrm{C}$, toujours avec des échantillons polycristallins et des pressions d'oxygène comprises entre $5 \times 10^{-5}$ torr et $1,5 \times 10^{-4}$ torr. Les courbes obtenues sont reportées dans la figure 6 , exceptée la courbe à $9 \times 10^{-5}$ torr qui était représentée figure 5 .

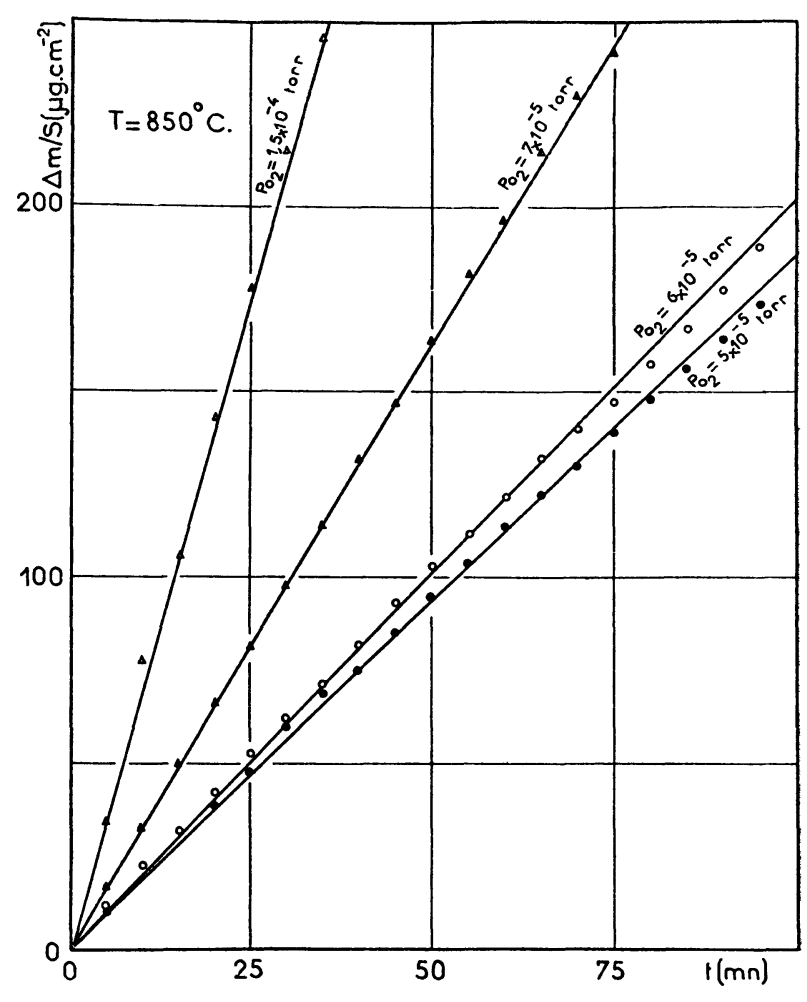

FIG. 6.

En portant le lagorithme de la constante de vitesse en fonction du lagorithme de la pression on obtient une droite comme le montre la figure 7 et le coefficient $n$ dans la relation:

$$
k=\operatorname{Cte}\left(p_{\mathrm{O}_{2}}\right)^{n}
$$

est très voisin de 1 . Il est en fait plus proche de 1,2 aux températures élevées.

c. Influence de l'orientation cristalline. - La vitesse de la réaction dépend en réalité de façon importante de l'orientation de la surface du métal sous-jacent. Afin de déterminer l'influence de ce facteur nous avons été amenés à réaliser des oxydations avec des cristaux uniques de niobium préparés sous ultra-vide, par la Société Alcatel que nous sommes heureux de remercier ici.

La figure 8 montre les courbes cinétiques obtenues à $800^{\circ} \mathrm{C}$ sous une pression d'oxygène de $9 \times 10^{-5}$ torr avec des cristaux d'orientations (100) et (111). On 


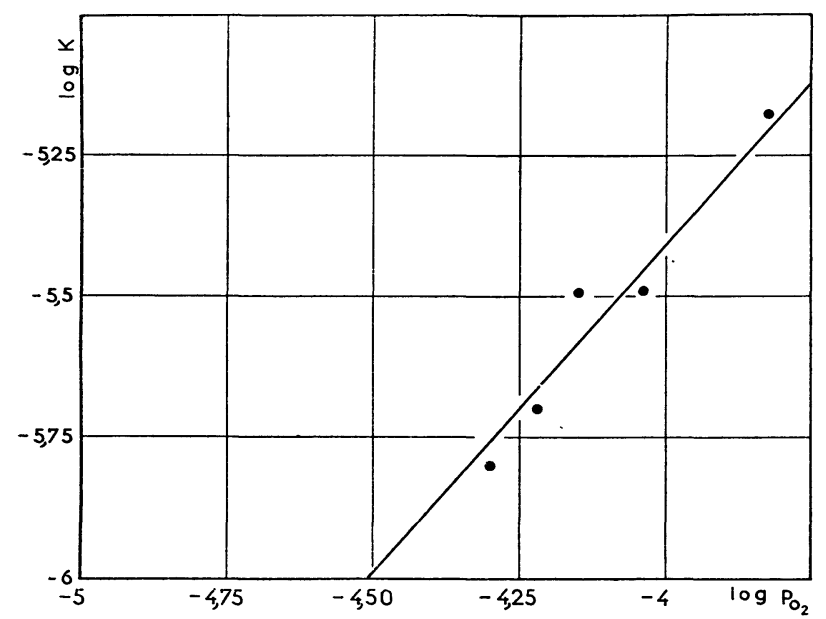

FIG. 7.

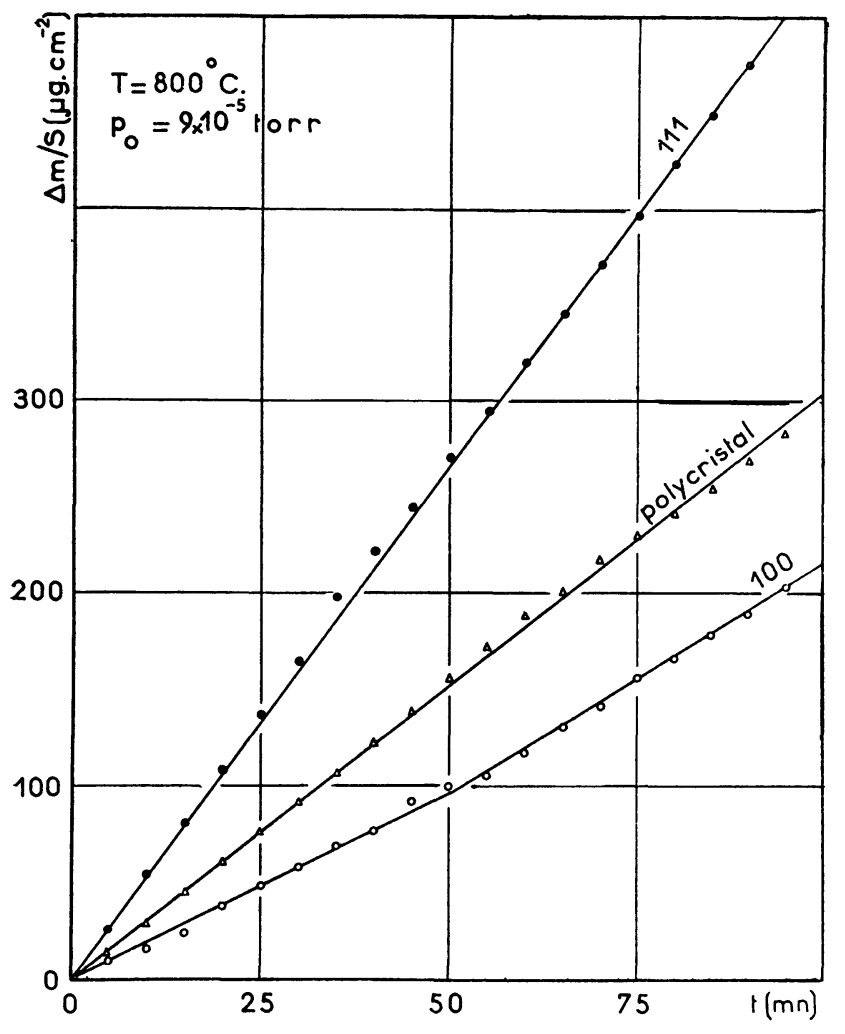

FIG. 8.

notera que la face (100) s'oxyde à une vitesse beaucoup plus faible que la face (111). De plus, un échantillon polycristallin a dans ces mêmes conditions un comportement intermédiaire entre ceux des deux orientations précédentes.

3. INTERPRÉTATION DES RÉSUltats. - Afin de donner une interprétation des résultats précédents tenant compte d'une part des deux valeurs de l'énergie d'activation et d'autre part de la quasi-proportionnalité de la vitesse à la pression d'oxygène, à une même température il était intéressant de déterminer le rendement de la réaction, c'est-à-dire le rapport du nombre de molécules fixées au nombre de molécules frappant la surface.
Le nombre de molécules fixées nous est donné directement par la constante de vitesse et le nombre de molécules frappant l'unité de surface par la théorie cinétique des gaz, celui-ci est égal à :

$$
\frac{p}{(2 \pi m k \mathrm{~T})^{1 / 2}}
$$

$p$ est la pression d'oxygène.

$m$ la masse de la molécule.

$k$ la constante de Boltzmann.

Admettons une vitesse d'adsorption $u$ de la forme suivante :

$$
u=\frac{\sigma \cdot p}{\sqrt{2 \pi m k \mathrm{~T}}} \cdot f(\theta) \cdot \exp \left(\frac{-E}{\mathrm{RT}}\right) .
$$

Dans cette expression $E$ est l'énergie d'activation du processus : $\sigma$ est le coefficient de condensation et représente la probabilité de collision entre une particule de l'adsorbat et un site d'adsorption de la surface de l'adsorbant; $f(\theta)$ est une fonction du taux de recouvrement $\theta$ de la surface.

Les produits $\sigma \cdot f(\theta)$ ont été calculés à la pression de $9 \times 10^{-5} \mathrm{~mm}$ de $\mathrm{Hg}$ d'oxygène et à diverses températures, en tenant compte de la vitesse de fixation des molécules $u$ déterminée expérimentalement.

Les résultats sont les suivants :

$\begin{array}{rccc}\mathrm{t}{ }^{\circ} \mathrm{C} & \mathrm{T}{ }^{\circ} \mathrm{K} & u \text { (molécules/s) } & \sigma . f(\theta) \\ - & - & - & - \\ 700 & 973 & 1,10 \times 10^{15} & 0,29 \\ 800 & 1073 & 1,28 \times 10^{15} & 0,30 \\ 1000 & 1273 & 1,49 \times 10^{15} & 0,31\end{array}$

$\mathrm{Si}$ on admet que le coefficient de condensation est voisin de 1 dans les conditions de température indiquées, le facteur $f(\theta)$ est de l'ordre de 0,3 , c'est-à-dire que $1 / 3$ de la surface interviendrait pour l'adsorption.

Un calcul analogue effectué pour des températures inférieures à $700^{\circ} \mathrm{C}$ pour lesquelles l'énergie d'activation trouvée est de $26 \mathrm{kcal}$ environ conduirait à des valeurs de $\sigma f(\theta)$ bien supérieures à l'unité (de l'ordre de 100). Ceci montre que l'on ne peut plus utiliser ici l'expression qui avait été indiquée en théorie pour la vitesse $u$ de fixation de l'oxygène.

En définitive, à la suite de ces considérations et de l'ensemble de nos résultats, nous proposons le schéma réactionnel suivant :

a. L'adsorption physique des molécules $\mathrm{O}_{2}$ est le phénomène le plus lent conditionnant la cinétique, aux températures les plus élevées considérées dans notre travail, c'est-à-dire entre 700 et $950^{\circ} \mathrm{C}$. Cette adsorption physique est ici un phénomène faiblement activé (3 $\mathrm{kcal} / \mathrm{mole})$ et s'effectue selon la réaction :

$$
\mathrm{O}_{2} \text { (gaz) } \rightarrow \mathrm{O}_{2} \text { (physisorbé) . }
$$

La valeur du produit $\sigma f(\theta)$ montre qu'il doit se former un complexe activé mobile à la surface du métal et l'énergie d'activation $W_{1}$ peut s'interpréter facilement en admettant 2 degrés de translation parallèlement à la surface du métal et un degré de 
rotation autour d'un axe perpendiculaire. Si l'on prend pour chaque degré de liberté une énergie moyenne égale à $1 / 2 \mathrm{RT}$ par mole on voit que le complexe activé nécessite une énergie égale à $3 / 2 \mathrm{RT}$, soit à $1000^{\circ} \mathrm{K}$ $3 \mathrm{kcal}$, ce qui correspond à la valeur $W_{1}$ trouvée pour l'énergie d'activation à haute température. En conséquence, seules les molécules d'oxygène possédant cette énergie pourront être physisorbées, ce phénomène nécessitant leur réarrangement en surface.

On peut admettre que cette adsorption physique est faiblement exothermique et la variation d'enthalpie correspond à la quantité de chaleur $q_{p}$.

b. Si l'adsorption chimique est le phénomène régulateur nous devons écrire la suite des réactions :

$$
\begin{gathered}
\mathrm{O}_{2} \text { (gaz) } \rightleftarrows O_{2} \text { (physisorbé) } \\
\mathrm{O}_{2} \text { (physisorbé) }+2 \text { sites } \rightarrow O \text { (chimisorbé) }+ \\
+O \text { (chimisorbé) } .
\end{gathered}
$$

On voit que la vitesse de cette dernière réaction est proportionnelle à la concentration en oxygène adsorbé physiquement. Si la chimisorption est un phénomène nettement moins rapide que l'adsorption physique, on peut admettre en première approximation que la concentration en oxygène physisorbé est essentiellement donnée par la première réaction qui correspond dans ce cas à un équilibre.

Nous admettrons que ce schéma réactionnel est celui qui correspond à la réaction aux températures inférieures à $700^{\circ} \mathrm{C}$ et tout au moins entre 700 et $550^{\circ} \mathrm{C}$. L'énergie d'activation est ici plus délicate à interpréter et il est nécessaire de faire intervenir une représentation de type analogue à celle proposée pour les courbes d'énergie potentielle par Lennard-Jones.

La figure 9 montre l'aspect des courbes d'énergie potentielle qu'on peut supposer pour les systèmes $\mathrm{Nb}+2 \mathrm{O}$ d'une part et $\mathrm{Nb}+\mathrm{O}_{2}$ d'autre part. $\mathrm{On}$ représente en fait l'énergie potentielle en fonction de la distance à la surface du métal. On constate que les courbes se coupent en un point $M$ et l'énergie d'activation de chimisorption correspond à l'ordonnée $\mathbf{C M}$ et a pour valeur $W_{2}=26 \mathrm{kcal} / \mathrm{mole}$. L'énergie de

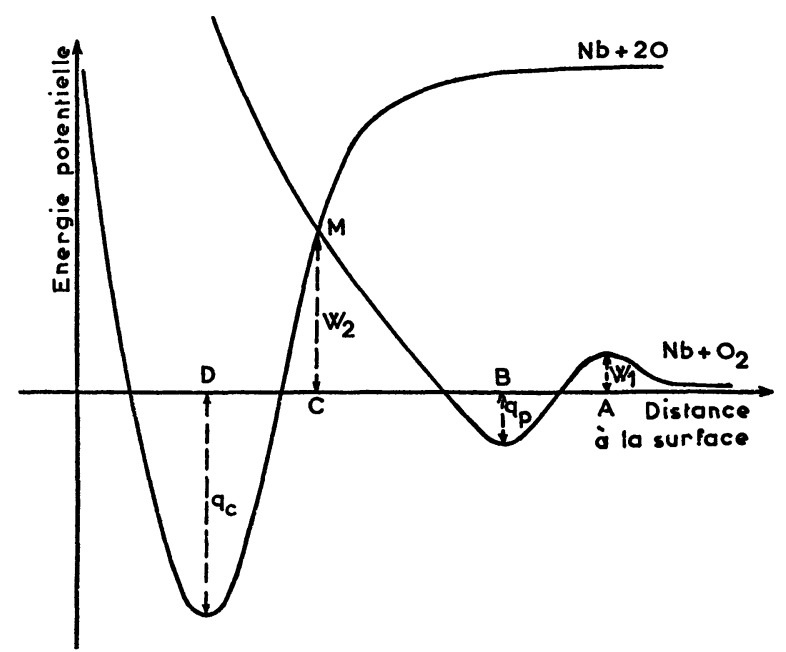

Fig. 9. - Courbes d'énergie potentielle pour les systèmes $\mathrm{Nb}+2 \mathrm{O}$ et $\mathrm{Nb}+\mathrm{O}_{2}$. chimisorption est définie par la valeur $q_{c}$. On peut se rendre compte que la valeur de $W_{2}$ est difficile à prévoir.

Le schéma proposé rend bien compte de nos résultats expérimentaux; ce n'est bien entendu pas le seul et on pourrait également envisager que le premier phénomène à haute température est une chimisorption peu activée et très exothermique et le second phénomène une chimisorption plus activée et moins exothermique (par exemple une chimisorption sur une mince couche d'oxyde). Ces deux chimisorptions s'expliqueraient alors selon les schémas proposés par Ehrlich [5]. Il semble toutefois que la valeur de l'énergie d'activation du premier phénomène soit plutôt en faveur d'une physisorption, comme nous le supposons.

III. Phénomène de germination. - 1. INFLUENCE DE L'ORIENTATION CRISTALLINE SUR LA CINÉTIQUE. L'étude du phénomène de germination à la platine chauffante a été réalisée en détail mais nous n'insisterons pas ici sur les résultats obtenus. Par contre, des résultats cinétiques qui concernent l'oxydation de cristaux uniques de niobium de diverses orientations, seront décrits.

Des cinétiques d'oxydation ont été réalisées en effet avec une balance de torsion du type Gulbransen à $900^{\circ} \mathrm{C}$ et $2 \times 10^{-4}$ torr. La figure 10 représente les courbes $\Delta m / S$ en microgrammes par $\mathrm{cm}^{2}$ en fonction $\mathrm{du}$ temps en minute, pour des orientations (110), (100) et (111) ainsi que pour un échantillon polycristallin. On peut noter que pour les conditions choisies ces courbes sont soit des droites, soit formées de deux segments de droite. La vitesse de prise de masse est

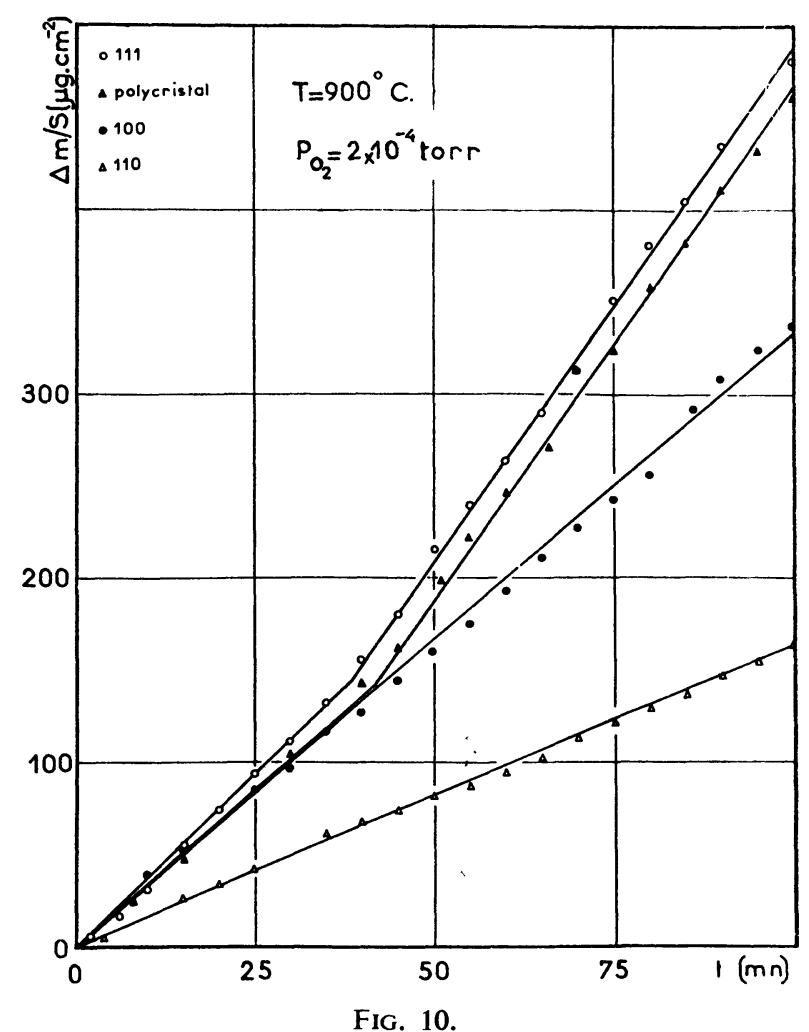


plus faible pour le plan (110) et croît dans l'ordre (110), (100), (111). La courbe (111) présente une cassure et après un certain temps la réaction devient plus rapide. Il en est de même pour l'échantillon polycristallin, ce qui n'est pas surprenant puisque sur ce dernier la majeure partie des orientations correspond à des faces (111).

Nous essayons actuellement d'interpréter ce compor- tement des cristaux uniques. La difficulté ici est de distinguer le phénomène d'absorption qui accompagne sans aucun doute celui de germination.

2. NATURE DES GERMES. - La nature des germes a été déterminée au diffractomètre pour diverses conditions de température et de pression à la fois sur échantillons polycristallins et sur cristaux uniques. Les résultats obtenus sont les suivants :

$T=1200^{\circ} \mathrm{C} \quad P_{\mathrm{O}_{2}}=8 \times 10^{-3}$ torr $\quad t=3^{\prime}$

les gros germes formés sont constitués des oxydes $\mathrm{NbO}$ et $\mathrm{NbO}_{2}$

\begin{tabular}{|c|c|c|c|c|}
\hline$T=1000^{\circ} \mathrm{C}$ & $\begin{array}{l}P_{O_{2}}= \\
P_{O_{2}}= \\
P_{O_{2}}=\end{array}$ & $\begin{array}{l}2 \times 10^{-3} \text { torr } \\
8 \times 10^{-4} \text { torr } \\
5 \times 10^{-3} \text { torr }\end{array}$ & $\begin{array}{l}t=5^{\prime} \text { et } 15^{\prime} \\
t=1 \text { h } 30 \\
t=3^{\prime} \\
t=1,5^{\prime}\end{array}$ & $\begin{array}{l}\mathrm{NbO} \\
\mathrm{NbO}_{2} \\
\mathrm{NbO}+\mathrm{NbO}_{2} \text { petits et gros germes } \\
\mathrm{NbO}\end{array}$ \\
\hline$T=\underset{\text { (monocristaux) }}{900^{\circ} \mathrm{C}}$ & $P_{O_{2}}=$ & $2 \times 10^{-4}$ torr & $t=100^{\prime}$ & $\mathrm{NbO}$ et $\mathrm{NbO}_{2}$ \\
\hline
\end{tabular}

les plans considérés ont été (100), (110) et (111). Les oxydes sont ici fortement épitaxiques et des raies fortes caractéristiques n'apparaissent pratiquement pas.

$\begin{array}{llll}T=850^{\circ} \mathrm{C} & P_{\mathrm{O}_{2}}=2 \times 10^{-4} \text { torr } & t=105^{\prime} & \mathrm{NbO}, \text { peu de } \mathrm{NbO}_{2} \\ T=700^{\circ} \mathrm{C} & P_{\mathrm{O}_{2}}=2 \times 10^{-4} \text { torr } & t=125^{\prime} & \mathrm{NbO} \text {, peu de } \mathrm{NbO}_{2} \\ T=650^{\circ} \mathrm{C} & P_{\mathrm{O}_{2}}=1,5 \times 10^{-4} \text { torr } & & \mathrm{NbO} \text { uniquement } \\ T=676^{\circ} \mathrm{C} & P_{\mathrm{O}_{2}}=1,5 \times 10^{-4} \text { torr } & t=135^{\prime} & \mathrm{NbO} \text { et peut-être } \mathrm{NbO}_{2}\end{array}$

En conclusion de cette étude sur la nature des germes ; on peut dire que ceux-ci sont constitués à haute température et sous basse pression d'oxygène, essentiellement des oxydes $\mathrm{NbO}$ et $\mathrm{NbO}_{2}$. $\mathrm{NbO}$ se forme en premier et d'autant plus facilement que la pression est plus basse. Ceci est en accord en général avec les résultats d'Inouye [3].

IV. Couches continues d'épaisseur notable. - Une étude cristallographique et une étude micrographique ont été conduites de façon parallèle.

1. AsPeCts MiCrographiQues. - Nous indiquerons ici quelques aspects d'échantillons oxydés dans différentes conditions.

La pression choisie a été 5 torr. L'aspect d'un échantillon oxydé pendant $30 \mathrm{mn}$ à $750^{\circ} \mathrm{C}$ est indiqué figure 11 .

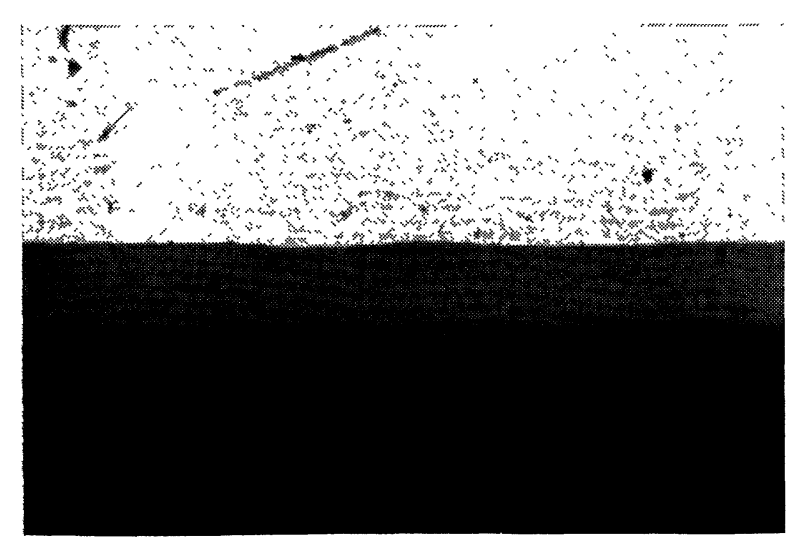

Fig. 11. - Coupe effectuée sur un échantillon oxydé à $750^{\circ} \mathrm{C}$ sous 5 torr pendant $30 \mathrm{mn}$.
Des coupes micrographiques ont été également réalisées sur des échantillons oxydés à $890^{\circ} \mathrm{C}$ pendant des temps de $15 \mathrm{mn}, 1 \mathrm{~h}$ et $3 \mathrm{~h}$ et sont représentées sur les figures 12,13 et 14 .

A $890^{\circ} \mathrm{C}$, au bout de $15 \mathrm{mn}$ l'échantillon présente un aspect noirâtre; après $1 \mathrm{~h}$ et $3 \mathrm{~h}$ il est recouvert d'une couche d'oxyde blanc très dure et très friable. Sur la figure 13, on voit les deux couches d'oxyde qui sont nettement séparées. Dans le cas de la coupe correspondant à la figure 14, les écailles blanches ont été enlevées et il est facile de voir que l'interface métal-oxyde s'est modifiée au cours du temps et ne présente plus l'aspect rectiligne.

2. IDENTIFICATION DES OXYDES AU DIFFRACTOMÈTRE. - a. Rappel sur la structure des différents oxydes de niobium. - Il n'est pas inutile de rappeler ici les divers types d'oxyde de niobium et en parti-

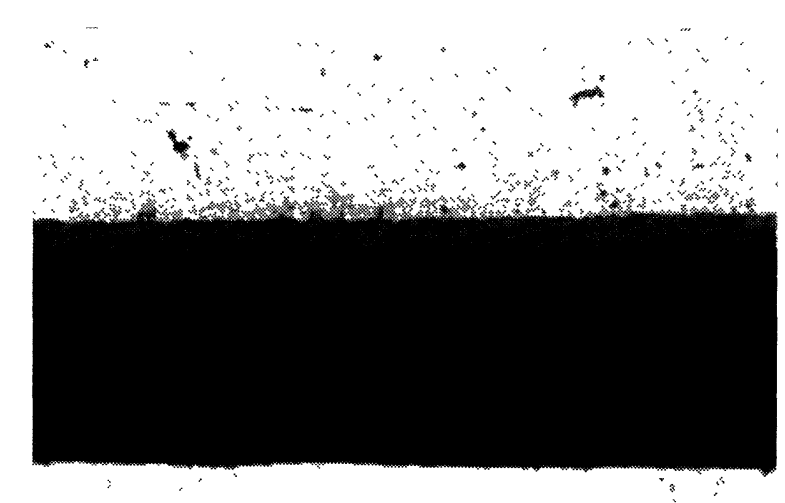

Fig. 12. - Coupe effectuée sur un échantillon oxydé à $890^{\circ} \mathrm{C}$ $p=5$ torr pendant $15 \mathrm{mn}$. 
culier de préciser leur dénomination car il existe une certaine confusion dans la littérature à ce sujet.

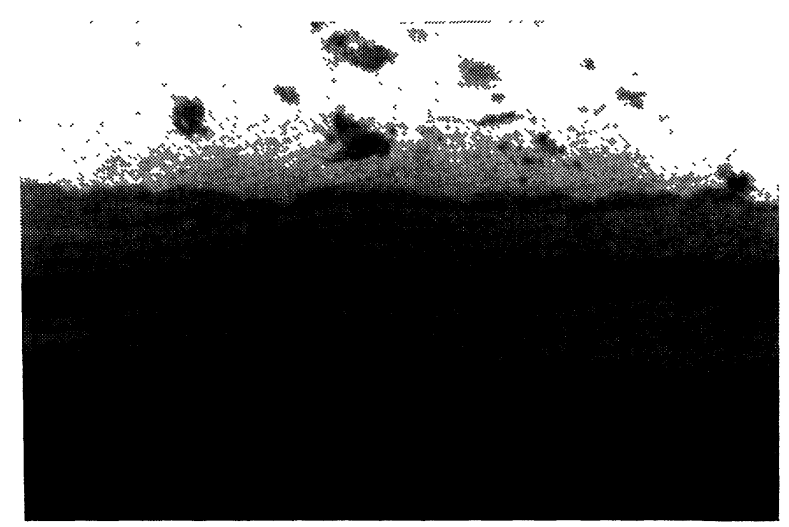

Fig. 13. - Coupe effectuée sur un échantillon oxydé à $890^{\circ} \mathrm{C}$ $p=5$ torr pendant $1 \mathrm{~h}$.

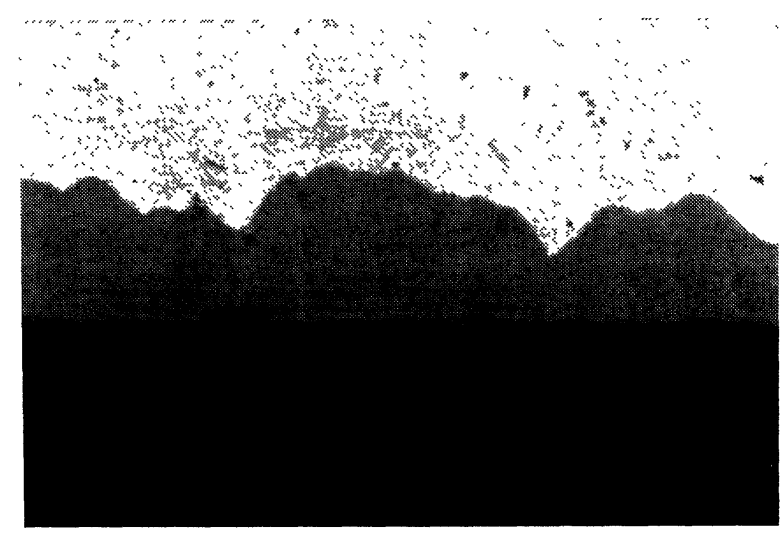

FIG. 14. - Coupe effectuée sur un échantillon oxydé à $8900^{\circ} \mathrm{C}$ $p=5$ torr pendant $3 \mathrm{~h}$.

$\mathrm{NbO}_{x}$ est une phase quadratique dont la structure est en relation avec celle du métal. Ses paramètres sont : $a=3,39 \AA$ et $c=3,27 \AA$ (paramètre de $\mathrm{Nb}=3,30 \AA)$. Ses raies caractéristiques sont très proches de celle du niobium et il est donc difficile de le mettre en évidence. Brauer [6] a montré qu'il ne se forme qu'à des températures comprises entre 300 et $450^{\circ} \mathrm{C}$.

$\mathrm{NbO}_{z}$ est aussi une phase quadratique isomorphe de la combinaison avec l'oxygène du Tantale, $\mathrm{TaO}_{z}$. Il a été mis en évidence par Norman [2] à basse pression d'oxygène entre 400 et $500{ }^{\circ} \mathrm{C}$.

$\mathrm{NbO}$ possède la structure de $\mathrm{NaCl}$.

$\overline{\mathrm{NbO}}_{2}$ est du type rutile.

Ces deux derniers oxydes ont été surtout observés à basse pression, et nous avons vu que ce sont eux qui constituent les germes.

$\mathrm{Nb}_{2} \mathrm{O}_{5}$ en ce qui concerne cet oxyde, il existe de nombreuses données en désaccord les unes avec les autres. Ces désaccords semblent provenir des conditions de préparation différentes suivant les auteurs et d'une mauvaise définition de la composition de l'oxyde.

Des divergences existent également au sujet de la dénomination des différentes phases.
Pour Goldschmidt [7] $\mathrm{Nb}_{2} \mathrm{O}_{5}$ existe sous 3 formes :

$\alpha \mathrm{Nb}_{2} \mathrm{O}_{5}$ : variété métastable dite de basse $T$ (jusqu'à $900^{\circ}$ ).

$\beta \mathrm{Nb}_{2} \mathrm{O}_{5}$ : variété stable entre 600 et $1250^{\circ} \mathrm{C}$.

$\gamma \mathrm{Nb}_{2} \mathrm{O}_{5}$ : variété stable au-dessus de $1250^{\circ} \mathrm{C}$.

Nous avons adopté cette dénomination dans notre travail. Il est intéressant toutefois de donner ici un aperçu des autres dénominations utilisées par divers auteurs.

Kofstad et également Terao [8] pensent qu'il existe une phase supplémentaire $\delta$ faiblement cristallisée entre 350 et $600^{\circ} \mathrm{C}$.

Cette phase aurait une structure pseudo-hexagonale.

De 600 à $900{ }^{\circ} \mathrm{C}$, cette phase $\delta$ se transforme rait en une phase $\gamma$ monoclinique. Toutefois, Goldschmidt [7], Brauer [6] et Holtzberg [9] considèrent qu'il existe seulement une même phase entre 350 et $900^{\circ} \mathrm{C}$ environ.

De 900 à $1100^{\circ}$ Kofstad et Brauer [6] trouvent une autre phase monoclinique qu'ils appellent respectivement $\beta$ et $M$.

De 1100 à $1200^{\circ} \mathrm{C}$, une nouvelle phase est appelée $\alpha$ par Kofstad et $H$ par Brauer.

Goldschmidt pense que les phases $\beta$ et $\alpha$ de Kofstad, c'est-à-dire $M$ et $H$ de Brauer n'ont pas de différence cristallographique et il admet qu'il n'existe qu'une seule phase monoclinique $\beta$ dans le domaine 900 à $1250^{\circ} \mathrm{C}$.

b. Appareillage utilisé et résultats. - L'appareillage à Rayons $\mathrm{X}$ utilisé comprend :

- un générateur stabilisé,

- une baie d'alimentation,

- une sonde à compteur proportionnel scellé.

Nous avons en général utilisé une anticathode de cuivre. Le zéro du goniomètre était réglée à $0,01^{\circ}$ près à l'aide de la raie du silicium.

Les résultats obtenus concernant la nature des oxydes constituant les couches épaisses peut se résumer comme suit :

En dessous de $800^{\circ} \mathrm{C}$ environ, on observe essentiellement la phase $\mathrm{Nb}_{2} \mathrm{O}_{5} \alpha$ de Goldschmidt. C'est ce que montrent les indications ci-dessous pour des échantillons oxydés respectivement à $650^{\circ} \mathrm{C}$ et $730^{\circ} \mathrm{C}$, un temps inférieur à $1 \mathrm{~h} 30$ et une pression d'oxygène égale à 5 torr.

$\begin{array}{ccc}650^{\circ} \mathrm{C} & 2 \theta & d(\AA) \\ - & - & - \\ & 22,70 & 3,92 \\ & 25,70 & 3,46 \\ 730^{\circ} \mathrm{C} & 28,20 & 3,15 \\ & 36,60 & 2,45 \\ & 22,00 & 3,95 \\ & 25,70 & 3,47 \\ & 28,15 & 3,16 \\ & 36,70 & 2,46 \\ & 42,80 & 2,12\end{array}$


A $890^{\circ} \mathrm{C}$ par contre les diagrammes de Rayons $\mathrm{X}$ d'échantillons oxydés $15 \mathrm{mn}, 30 \mathrm{mn}$ et $2 \mathrm{~h}$ permettent de conclure à la formation d'oxyde $\mathrm{Nb}_{2} \mathrm{O}_{5} \beta$ selon la notation de Goldschmidt. Cet oxyde est formé d'écailles blanches très dures du côté de l'atmosphère et noires du côté du métal.

L'existence des oxydes $\mathrm{NbO}$ et $\mathrm{NbO}_{2}$ n'est pas exclue dans la couche interne mais elle est très diffcile à prouver du fait que les raies caractéristiques de ces oxydes peuvent être interprétées comme appartenant à la phase $\beta$ et en tout cas si ces oxydes existent ils sont en faible quantité et ne présentent pas de couches séparées. Des abrasions successives ont été faites sur un échantillon oxydé à $890^{\circ} \mathrm{C}$ mais, même dans ce cas, $\mathrm{NbO}$ et $\mathrm{NbO}_{2}$ n'ont pu être mis en évidence avec certitude. De toute façon la phase $\mathrm{Nb}_{2} \mathrm{O}_{5}$ $\beta$ constitue la majeure partie de l'oxyde.

Si la transformation $\alpha \rightarrow \beta$ peut avoir un rôle important au voisinage de $850^{\circ} \mathrm{C}$ il semble bien toutefois que la variation de stoéchiométrie de l'oxyde qui se traduit par une différence de coloration et surtout de compacité joue un rôle essentiel vis-à-vis de la cinétique de la réaction. Il est bien certain que l'apparition de l'oxyde friable blanc conduit à l'oxydation catastrophique du métal.

Conclusions. - Nous pensons avoir apporté dans ce travail un ensemble de résultats nouveaux concernant les aspects fondamentaux de la réaction d'oxydation du niobium.

En ce qui concerne la formation des films minces interférentiels nous avons pu sur échantillons polycristallins préciser la cinétique de formation de ces films et sur monocristaux montrer l'anisotropie réactionnelle très grande qui existe dans ce cas.

L'adsorption de l'oxygène a été étudiée sous son aspect cinétique en fonction de facteurs température, pression et orientation cristalline et nous avons pu distinguer deux phénomènes différents en fonction de la température, l'un pouvant être l'adsorption physique des molécules $\mathrm{O}_{2}$ aux températures les plus élevées avec une faible énergie d'activation $(3 \mathrm{kcal} / \mathrm{mole}$, l'autre la chimisorption et l'énergie d'activation est ici plus élevée (26 kcal/mole). L'adsorption physique est fonction de l'orientation cristalline du métal.

La germination a été étudiée sous son aspect cinétique sur monocristaux et échantillons polycristallins. Elle est, elle aussi, fonction de l'orientation du métal mais les phénomènes sont compliqués par l'adsorption directe à la surface du métal qui a lieu simultanément. Dans les conditions choisies le plan (111) est celui de la réactivité maximale. La nature des germes a également été déterminée et consiste en oxydes $\mathrm{NbO}$ et $\mathrm{NbO}_{2}$.

En ce qui concerne la formation des couches continues d'épaisseur notable nous avons pu vérifier qu'en dessous de $800^{\circ} \mathrm{C}$ il se forme essentiellement la phase $\alpha \mathrm{Nb}_{2} \mathrm{O}_{5}$ de Goldschmidt et à $890^{\circ} \mathrm{C}$ la phase $\beta$ $\mathrm{Nb}_{2} \mathrm{O}_{5}$. Cette dernière a des propriétés très différentes en fonction de sa stoéchiométrie et il convient de souligner une fois de plus que l'oxyde blanc poreux et friable est responsable pour une large part des médiocres qualités de résistance à l'oxydation du niobium.

Nous pensons à la suite de ces observations que le niobium présente un très grand intérêt sur le plan fondamental par suite de la réactivité très particulière, mais il est bien évident que sa protection qui est le souci majeur des utilisateurs est le but essentiel à rechercher. Il ne faut pas se dissimuler, qu'elle paraît présenter encore de très grandes difficultés.

Ces recherches toujours en cours ont été poursuivies au Laboratoire de Chimie des Solides à la Faculté des Sciences d'Orléans et au Centre de la Combustion et de la Chimie des Hautes Températures du C. N. R. S. à Orléans, en liaison avec le laboratoire du Professeur Bénard à l'Ecole Nationale Supérieure de Chimie de Paris.

Nous sommes heureux d'exprimer ici notre vive gratitude à la Direction des Recherches et Moyens d'Essais qui a financé ce travail.

Nous tenons également à remercier ici, Madame Bajard qui a apporté sa collaboration technique à ce travail.

\section{Bibliographie}

[1] Hurlen (T.), J. Inst. Metals, 1960-1961, 89, 273.

[2] Norman (N.), J. Less Common Metals, 1962, 4, 124.

[3] INOUYE, Columbium Metallurgy, Metallurgical Society Conference (AIME), 1960, 10, édité par Douglass (D. L.) et Kunz (F. W.), Interscience, New York, 649.

[4] Kofstad (P.) et EspeviK (S.), J. Electrochem. Soc., 1965, 112- 153.
[5] Ehrlich (G.), J. Chemical Physics, 1959, 31, 1111-1126.

[6] Brauer, $Z$. anorg. allgem. chemie, 1941, 243, 1-13.

[7] Goldschmidt (H. J.), J. Inst. Metals, 1958-1959, 87, 235.

[8] Terao, Japanese Journal of Applied Physics, 1963, 2, 156.

[9] Holtzberg, Reisman, Bery, Berkenblit, J. Amer. Chem. Soc., 1957, 79, 2039-2043. 